
Brasileira 26: 068-073.

\title{
Macronutrientes em cultivares de gérbera sob dois níveis de fertirrigação ${ }^{1}$
}

\author{
Fernanda Ludwig'; Dirceu Maximino Fernandes ${ }^{2 ; 3}$; Poliana RD Mota ${ }^{2 ;}$ Roberto L Villas Bôas;3 $^{2 ; 3}$ \\ ${ }^{2}$ UNESP-FCA, Depto. Recursos Naturais, C. Postal 237, 18610-307 Botucatu-SP; ${ }^{3 B o l s i s t a ~ C N P q ; ~ f l u d w i g @ f c a . u n e s p . b r ~}$
}

\begin{abstract}
RESUMO
A gérbera de vaso é uma planta ornamental conhecida mundialmente; entretanto, dispõem-se de reduzido volume de informações sobre suas técnicas de cultivo, em especial a nutrição e adubação, fatores essenciais para ganhos na qualidade produtiva. O presente trabalho teve por objetivo avaliar o teor e o acúmulo de macronutrientes em quatro cultivares de gérbera sob dois níveis de fertirrigação. O experimento foi conduzido em casa de vegetação, de maio a julho de 2006, na UNESP em Botucatu. O mesmo foi instalado em blocos casualizados e constituído de um fatorial $4 \times 2$, sendo 4 cultivares de gérbera (Cherry, Golden Yellow, Salmon Rose e Orange) e 2 concentrações de solução nutritiva (50\% e 100\%), correspondendo respectivamente às condutividades elétricas de 0,92 e $1,76 \mathrm{dS} \mathrm{m}^{-1}$, no período vegetativo e 1,07 e $2,04 \mathrm{dS} \mathrm{m}^{-1}$, no reprodutivo. Os nutrientes foram aplicados via fertirrigação, realizada manualmente, uma vez ao dia. Ao final dos períodos vegetativo e reprodutivo foram quantificados no tecido vegetal, os teores e os acúmulos dos macronutrientes. As demandas destes nutrientes foram diferenciadas entre as cultivares e a concentração da solução apresentou um efeito marcante no teor e acúmulo de N, P, Ca, Mg e $\mathrm{S}$ na planta. Os maiores acúmulos foram registrados no terço final do ciclo de produção, apresentando a seguinte ordem de absorção: $\mathrm{K}>\mathrm{N}>\mathrm{Ca}>\mathrm{Mg}>\mathrm{P}>\mathrm{S}\left(415,327,33,32,20 \mathrm{mg} \mathrm{planta}^{-1}\right)$.
\end{abstract}

Palavras-chave: Gerbera jamesonii, nutrição mineral, avaliação nutricional.

\begin{abstract}
Macronutrients in gerbera cultivars under two fertigation levels

Potted gerbera is known world-wide as an ornamental plant; althought there is a reduced volume of information on its cultivation, specially nutrition and fertilization, essential factors for profits in the productive quality. In the present work the content and accumulation of macronutrients was evaluated in four gerbera cultivars under two levels of fertigation. The experiment was carried out in a greenhouse, from May to July, 2006. The experiment was installed in randomized blocks and constituted of a $4 \times 2$ factorial scheme, being 4 gerbera cultivars (Cherry, Golden Yellow, Salmon Rose and Orange) and 2 concentrations of nutritive solution (50\% and $100 \%$ ), corresponding to the electrical conductivity of 0,92 and $1,76 \mathrm{dS} \mathrm{m}^{-1}$, during the vegetative period and 1,07 and 2,04 dS m $\mathrm{m}^{-1}$, during the reproductive one. The nutrients were applied through manual fertigation, once a day. By the end of the vegetative and reproductive periods, the contents and accumulation of macronutrients were quantified. The nutrients demand differed among the cultivars and the solution concentration presented an important factor in the content and accumulation of $\mathrm{N}, \mathrm{P}, \mathrm{Ca}, \mathrm{Mg}$ and $\mathrm{S}$ in the plant. The greatest accumulations was registered at the last third of the production cycle, presenting the following order of absortion: $\mathrm{K}>\mathrm{N}>\mathrm{Ca}>\mathrm{Mg}>\mathrm{P}>\mathrm{S}$ (415, 327, 33, 32, $20 \mathrm{mg} \mathrm{plant}^{-1}$ ).
\end{abstract}

Keywords: Gerbera jamesonii, mineral nutrition, nutritional evaluation.

\section{(Recebido para publicação em 12 de janeiro de 2007; aceito em 25 de fevereiro de 2008)}

\begin{abstract}
A gérbera (Gerbera jamesonii), família Asteraceae, originária do sul da África e Ásia, deu origem a inúmeros híbridos, que exibem diversas combinações de cores e formas em suas inflorescências, de grande interesse no mercado consumidor (Radice \& Marconi, 1998). A comercialização dessa espécie na América do Norte teve início em 1920, com o objetivo de produção de flores de corte. O desenvolvimento de cultivares para vaso iniciouse em 1980, no Japão, representando o maior avanço para a cultura (Rojers \& Tjia, 1990). Bellé (1998) relata que a espécie tem sido muito estudada como flor de corte, sendo que sua difusão como planta de vaso é recente, dispon-
\end{abstract}

do-se de reduzido volume de informações a respeito das técnicas de cultivo.

A cultura da gérbera é conhecida mundialmente pelo amplo número de cultivares disponíveis no mercado. De acordo com Chung et al. (2001), a cada ano são lançadas mais de 300 cultivares, as quais respondem de maneira diferenciada aos vários fatores de produção, dentre eles, a adubação.

O conhecimento da adubação e nutrição são fatores essenciais para ganhos na qualidade produtiva. Entretanto, no Brasil, estes se baseiam principalmente no empirismo ou nas recomendações de outros países, o que pode acarretar em desbalanços nutricionais. Segundo Kämpf (2000), a característica mais importante para que uma planta cumpra sua função ornamental é o seu aspecto saudável, atestando estar bem nutrida e hidratada.

São poucas as informações sobre a nutrição de gérbera citadas na literatura, sendo estas quase inexistentes no Brasil. Segundo Rojers \& Tjia (1990), muitos pesquisadores consideram que essa planta tem médio requerimento quanto aos macronutrientes, e recomendam proporções e quantidades de fertilizantes similares às usadas pela cultura do crisântemo. Camargo et al. (2004), ao estudarem Aster ericoides, também pertencente à família Asteraceae, apontam para a falta de pesquisas com relação a esta cultura e justificam a realização de estudos com-

\footnotetext{
${ }^{1}$ Parte da Dissertação do primeiro autor, apresentada a FCA/UNESP, para obtenção do título de Mestre em Agronomia (Horticultura).
} 
parativos com base em resultados já conhecidos para culturas floríferas da mesma família botânica.

A concentração de um nutriente na planta é indicador final de vários fatores que influenciam seu crescimento, sendo inquestionável a contribuição da análise foliar para avaliar a qualidade da produção agrícola e como alerta aos produtores quanto à necessidade de suplementação de nutrientes para aumentar ou melhorar a produção (Stringueta et al., 2003). O objetivo do presente trabalho foi avaliar o teor e o acúmulo dos macronutrientes em quatro cultivares de gérbera fertirrigada com duas soluções nutritivas, quantificando assim, suas necessidades nutricionais.

\section{MATERIAL E MÉTODOS}

O experimento foi conduzido de 03/ 05 a 03/07/06, em casa de vegetação da UNESP, município de Botucatu (SP), com coordenadas geográficas de $21^{\circ} 51^{\text {' }}$ de latitude sul, $48^{\circ} 26^{\prime}$ de longitude oeste e altitude média de $830 \mathrm{~m}$. A casa de vegetação apresentava estrutura de teto em arco, com plástico transparente, laterais de tela branca e pavimentada com concreto. Aárea total era de $168 \mathrm{~m}^{2}$, com 2,6 m de pé direito e 3,0 m de altura no vão central. O ambiente superior interno possuía malha termorefletora com $50 \%$ (Aluminet ${ }^{\circledR}$ ) manejada de acordo com a intensidade luminosa (Lux), permanecendo fechada das 10:30 às 16:00 horas e aberta nos demais horários, onde a intensidade luminosa era inferior a 50.000 Lux. A temperatura média dentro da casa de vegetação foi de $20,5^{\circ} \mathrm{C}$ e a e umidade relativa média do ar de 69\%.

O delineamento experimental adotado foi em blocos casualizados, empregando o esquema fatorial 4x2 (4 cultivares e 2 soluções nutritivas), em 5 repetições e 6 plantas por parcela. Os vasos foram espaçados de 30 em $30 \mathrm{~cm}$, totalizando uma área útil de $21,6 \mathrm{~m}^{2}$, com 14,4 m de comprimento e 1,5 m de largura. Os mesmos foram colocados sobre tijolos, dispostos no piso da estufa.

As cultivares utilizadas são provenientes da geração $F_{1}$ do grupo Festival, da empresa Sakata ${ }^{\circledR}$, de grande aceitação no mercado, sendo Cherry e
Golden Yellow, pertencentes à série Dark Eyes (centro escuro), e Salmon Rose e Orange, pertencentes à série Light Eyes (centro claro).

As soluções nutritivas constaram de uma referência padrão para gérbera de vaso, $100 \%$, utilizada pela empresa Steltenpool, e uma diluição em 50\% da mesma. A composição da solução 100\%, em mg dm³ ${ }^{-3}$, foi: 142,0 $\mathrm{NO}_{3}^{-} ; 101,5$ $\mathrm{NH}_{4}^{+}$; 105,0 K; 25,2 P; 51,3 Ca; 6,3 Mg; 28,0 S; 0,2 B; 0,3 Cu; 3,9 Fe; 1,4 Mn; 0,1 Mo e 0,3 Zn, no período vegetativo. Estas foram reformuladas quando a planta entrou na fase reprodutiva, aos 41 dias após a aclimatação (DAA), devido à mudança na demanda da cultura, tendo a seguinte composição em $\mathrm{mg} \mathrm{dm}^{-3}$ : 110,3 $\mathrm{NO}_{3}^{-}, 66,8 \mathrm{NH}_{4}^{+} ; 285,0 \mathrm{~K} ; 56,6$ P; 26,3 Ca; 17,18 Mg; 76 S; 0,4 B; 0,4 $\mathrm{Cu}$; 4,4 Fe; 1,7 Mn; 0,1 Mo e 0,5 Zn. Para a solução 50\%, a quantidade dos nutrientes foi reduzida pela metade, em ambos os períodos.

As condutividades elétricas no período vegetativo correspondentes às soluções 50\% e 100\% foram, respectivamente: 0,92 e 1,76 dS m-1. No período reprodutivo, passaram para 1,07 e 2,04 $\mathrm{dS} \mathrm{m} \mathrm{m}^{-1}$. O pH das soluções nutritivas foi ajustado para 5,0 no momento de preparo da solução, com adição de ácido fosfórico (52\% $\left.\mathrm{P}_{2} \mathrm{O}_{5}\right)$, em ambos os períodos. As soluções preparadas foram estocadas em recipientes com capacidade de $90 \mathrm{~L}$.

Para a instalação do experimento foram utilizadas mudas de Gerbera jamesonii, para cultivo em vaso, com tratamento prévio de aclimatação, durante 30 dias. As plantas foram conduzidas em vasos número 15 (15 cm de diâmetro e 11,5 cm de altura, com volume de 1,3 L), preenchidos com substrato composto de $70 \%$ de casca de pinus fina e 30\% de terra de subsolo.

Os nutrientes foram fornecidos às plantas por meio da fertirrigação, aplicada manualmente, uma vez ao dia. A lâmina de irrigação correspondeu à quantidade de água requerida, a fim de elevar a umidade do substrato contido no vaso ao valor correspondente à condição de máxima retenção (Fanela et al., 2006). A quantidade média de solução nutritiva fornecida às cultivares Golden Yellow e Orange foi de $100 \mathrm{~mL}$ vaso $^{-1}$ $\mathrm{dia}^{-1}$ nos períodos vegetativo e reprodutivo, e de $100 \mathrm{~mL}$ vaso $^{-1} \mathrm{dia}^{-1}$ no período vegetativo e $150 \mathrm{~mL}$ vaso $^{-1} \mathrm{dia}^{-1}$ no período reprodutivo para Cherry e Salmon Rose. Assim, as duas primeiras cultivares receberam ao longo do ciclo 5,2 L de solução vaso ${ }^{-1}$ e as demais 5,9 $\mathrm{L}$ vaso $^{-1}$. Esta variação ocorreu devido às diferentes necessidades hídricas das cultivares.

A determinação do teor e acúmulo dos macronutrientes foi realizada ao final dos períodos vegetativo e reprodutivo. As plantas foram cortadas rente ao substrato, lavadas e postas para secar em estufa de ventilação forçada a $65^{\circ} \mathrm{C}$ por um período médio de 48 horas, até peso constante, seguido pela moagem em moinho do tipo "Willey".

A análise química do tecido vegetal seguiu a metodologia recomendada por Malavolta et al. (1997), para a determinação de N, P, K, Ca, Mg e S.

Os dados foram submetidos à análise de variância com teste $\mathrm{F}$. As médias foram comparadas pelo teste de Tukey a 5\% de probabilidade, quando os valores foram significativos, com uso do programa estatístico Sisvar. Procedeuse o desdobramento da interação entre cultivares e soluções nutritivas quando a análise de variância revelou efeito significativo.

\section{RESULTADOS E DISCUSSÃO}

As cultivares apresentaram comportamentos diferenciados em relação aos teores de nitrogênio (N). Aos 41 DAA, Orange, sem diferir de Golden Yellow, apresentou maior teor. No entanto, não houve diferença significativa entre as cultivares para o acúmulo de N (Tabela 1). Possivelmente, houve efeito de concentração deste nutriente nestas duas cultivares, pois seus valores de massa seca foram inferiores às demais (Tabela 2).

Aos 62 DAA houve efeito da interação, onde as cultivares responderam de maneira diferenciada na solução 50\%, tendo Golden Yellow apresentado o maior valor, superando estatisticamente Salmon Rose (Tabelas 1 e 3). Paradiso et al. (2003) ao avaliarem duas cultivares de gérbera, Brittani e Golden Serena, em dois níveis de solução nutritiva de 1,6 e 2,4 dS m ${ }^{-1}$, não encontra- 
Tabela 1. Teor ( $\mathrm{g} \mathrm{kg}^{-1}$ ) e acúmulo (mg planta ${ }^{-1}$ ) dos macronutrientes em plantas de gérbera, obtidos aos 41 e 62 DAA. Botucatu, UNESP, 2006.

\begin{tabular}{|c|c|c|c|c|c|c|c|c|c|c|c|c|}
\hline & \multicolumn{12}{|c|}{ Nutrientes } \\
\hline & \multicolumn{2}{|c|}{$\mathbf{N}$} & \multicolumn{2}{|c|}{$\mathbf{P}$} & \multicolumn{2}{|c|}{$\mathrm{K}$} & \multicolumn{2}{|c|}{$\mathrm{Ca}$} & \multicolumn{2}{|c|}{$\mathbf{M g}$} & \multicolumn{2}{|c|}{$\mathrm{S}$} \\
\hline & \multicolumn{12}{|c|}{ DAA } \\
\hline & 41 & 62 & 41 & 62 & 41 & 62 & 41 & 62 & 41 & 62 & 41 & 62 \\
\hline Cultivar (C) & \multicolumn{12}{|c|}{ g kg $^{-1}$} \\
\hline Cherry & $28 \mathrm{~b}$ & 28 & $1,6 \mathrm{~b}$ & 2,4 & $41 a b$ & $39 a$ & $10 \mathrm{~b}$ & $9 a$ & $2,9 \mathrm{~b}$ & $2,9 \mathrm{~b}$ & $1,5 \mathrm{~b}$ & 1,7 \\
\hline Golden Yellow & $32 a b$ & 29 & $2,4 a$ & 2,6 & $41 a b$ & $37 \mathrm{a}$ & $10 \mathrm{~b}$ & $10 \mathrm{a}$ & $2,6 \mathrm{~b}$ & $2,8 b$ & $1,5 b$ & 1,6 \\
\hline Salmon Rose & $28 \mathrm{~b}$ & 28 & $1,9 \mathrm{~b}$ & 2,7 & $38 \mathrm{~b}$ & $34 \mathrm{~b}$ & $12 a$ & $10 \mathrm{a}$ & 4,1 a & $3,7 \mathrm{a}$ & $1,7 a b$ & 2,1 \\
\hline Orange & $33 a$ & 29 & $2,0 \mathrm{~b}$ & 2,5 & $44 \mathrm{a}$ & $36 a b$ & $10 \mathrm{~b}$ & $7 \mathrm{~b}$ & $2,8 \mathrm{~b}$ & $2,3 \mathrm{c}$ & $1,8 \mathrm{a}$ & 1,8 \\
\hline \multicolumn{13}{|l|}{ Solução (S) } \\
\hline $50 \%$ & $28 \mathrm{~B}$ & 27 & $1,8 \mathrm{~B}$ & 2,3 & 42 & 37 & $10 \mathrm{~B}$ & $8,7 \mathrm{~B}$ & $2,9 \mathrm{~B}$ & $2,7 \mathrm{~B}$ & $1,6 \mathrm{~B}$ & 1,9 \\
\hline $100 \%$ & $32 \mathrm{~A}$ & 30 & $2,2 \mathrm{~A}$ & 2,8 & 40 & 36 & $11 \mathrm{~A}$ & $10,1 \mathrm{~A}$ & $3,3 \mathrm{~A}$ & $3,1 \mathrm{~A}$ & $1,7 \mathrm{~A}$ & 1,7 \\
\hline$C^{*} S$ & NS & * & NS & * & NS & NS & NS & NS & NS & NS & NS & ** \\
\hline CV (\%) & 11,6 & 7,2 & 17,1 & 10,9 & 11,6 & 6,9 & 8,8 & 14,1 & 10,5 & 12,0 & 12,4 & 11,0 \\
\hline Cultivar (C) & \multicolumn{12}{|c|}{ mg planta-1 } \\
\hline Cherry & 120 & 340 & 10 & 30 & 180 & $460 \mathrm{a}$ & 40 & $110 \mathrm{~b}$ & $10 \mathrm{~b}$ & $30 \mathrm{~b}$ & $6 \mathrm{~b}$ & $20 a b$ \\
\hline Golden Yellow & 120 & 310 & 10 & 30 & 160 & $390 a b$ & 40 & $110 b$ & $10 \mathrm{~b}$ & $30 \mathrm{~b}$ & $6 b$ & $17 \mathrm{~b}$ \\
\hline Salmon Rose & 140 & 350 & 10 & 40 & 200 & $440 a b$ & 60 & $140 \mathrm{a}$ & $20 a$ & $50 \mathrm{a}$ & $8 a$ & $26 a$ \\
\hline Orange & 140 & 310 & 10 & 30 & 190 & $370 \mathrm{~b}$ & 40 & $80 c$ & $10 \mathrm{~b}$ & $20 \mathrm{c}$ & $7 \mathrm{ab}$ & $18 \mathrm{~b}$ \\
\hline \multicolumn{13}{|l|}{ Solução (S) } \\
\hline $50 \%$ & $100 \mathrm{~B}$ & $300 \mathrm{~B}$ & $7 \mathrm{~B}$ & 27 & 180 & 400 & 40 & $90 \mathrm{~B}$ & 12 & $30 \mathrm{~B}$ & $6 \mathrm{~B}$ & 20 \\
\hline $100 \%$ & $200 \mathrm{~A}$ & $400 \mathrm{~A}$ & $10 \mathrm{~A}$ & 30 & 180 & 430 & 50 & $120 \mathrm{~A}$ & 16 & $40 \mathrm{~A}$ & $8 \mathrm{~A}$ & 20 \\
\hline$C^{*} S$ & NS & NS & NS & NS & NS & NS & * & NS & NS & NS & NS & NS \\
\hline CV (\%) & 22,3 & 16,0 & 34,6 & 21,3 & 24,7 & 14,7 & 18,3 & 16,7 & 19,1 & 16,3 & 27,1 & 22,2 \\
\hline
\end{tabular}

Valores seguidos pela mesma letra na coluna não diferem entre si pelo teste de Tukey a 5\%. C*S: interação entre cultivares e soluções nutritivas. NS: não significativo ao nível de 5\% de probabilidade.*: significativo a 5\% de probabilidade. **: significativo a 1\% de probabilidade. DAA: Dias após a aclimatação.

Tabela 2. Massa seca (g) em plantas de gérbera, obtida aos 41 e 62 DAA. Botucatu, UNESP, 2006.

\begin{tabular}{|c|c|c|c|}
\hline & \multicolumn{3}{|c|}{ Massa seca $\mathbf{g}$} \\
\hline & \multirow[t]{2}{*}{41 DAA } & \multicolumn{2}{|c|}{62 DAA } \\
\hline & & Solução $50 \%$ & Solução $100 \%$ \\
\hline \multicolumn{4}{|l|}{ Cultivar (C) } \\
\hline Cherry & $4,5 a b$ & $11,99 a b A$ & 11,84 a $A$ \\
\hline Golden Yellow & $3,9 \mathrm{~b}$ & $10,41 \mathrm{bc} \mathrm{A}$ & 11,90 a $A$ \\
\hline Salmon Rose & $5,2 \mathrm{a}$ & 13,38 a $A$ & 12,36 a $A$ \\
\hline Orange & $4,3 a b$ & $8,82 \mathrm{c} \quad \mathrm{B}$ & 12,06 a $A$ \\
\hline \multicolumn{4}{|l|}{ Solução (S) } \\
\hline $50 \%$ & 4,3 & & \\
\hline $100 \%$ & 4,7 & & \\
\hline $\mathrm{C}^{*} \mathrm{~S}$ & NS & \multicolumn{2}{|c|}{${ }^{*}$} \\
\hline $\mathrm{CV}(\%)$ & 21,5 & \multicolumn{2}{|c|}{13,65} \\
\hline
\end{tabular}

Valores seguidos pela mesma letra na coluna não diferem entre si pelo teste de Tukey a 5\%. C*S: interação entre cultivares e soluções nutritivas. NS: não significativo ao nível de $5 \%$ de probabilidade. ${ }^{*}$ : significativo a $5 \%$ de probabilidade. $* *$ : significativo a $1 \%$ de probabilidade. DAA: Dias após a aclimatação.

ram diferenças significativas entre elas para a absorção deste nutriente, ao final do período produtivo.

As plantas apresentaram maior teor e acúmulo de $\mathrm{N}$ quando submetidas à solução nutritiva $100 \%$, exceto para
Golden Yellow, aos 62 DAA. Paradiso et al. (2003), verificaram aumento na absorção deste elemento quando submetidas à solução com 2,4 dS m-1 em relação à solução com 1,6 dS m"-1. Entretanto, Zheng et al. (2004) não observaram diferenças no teor de $\mathrm{N}$ na aplicação via subirrigação de soluções com 100, 50, 25 e $10 \%$ de concentração, e condutividades elétricas de 1,7; 1,0; 0,5 e $0,2 \mathrm{dS} \mathrm{m}^{-1}$ respectivamente, indicando resultados superiores aos apontados na presente pesquisa, com uma média de $35 \mathrm{~g} \mathrm{~kg}^{-1}$.

Os valores de $\mathrm{N}$ obtidos, encontramse dentro ou pouco acima da faixa adequada de 27 a $31 \mathrm{~g} \mathrm{~kg}^{-1}$, proposta por Mercurio (2002). Valores inferiores foram registrados por Bellé (1998), de 18 a 26,3 $\mathrm{g} \mathrm{kg}^{-1}$, e por Savvas \& Gizas (2002), de $21 \mathrm{~g} \mathrm{~kg}^{-1}$, em folhas novas completamente expandidas.

Considerando o ciclo da gérbera, nota-se que até os $41 \mathrm{DAA}$, metade da quantidade de $\mathrm{N}$ havia sido incorporada à planta e que num prazo de 21 dias houve duplicação desta. Isto significa o quanto a adubação nitrogenada na fase reprodutiva é importante para a cultura, especialmente pela quantidade total acumulada ao final do ciclo.

Teores mais elevados de fósforo (P) 
foram observados em Golden Yellow, aos 41 DAA, porém sem correspondência em termos de acúmulo (Tabela 1), devido ao efeito de concentração. O comportamento das cultivares, para o teor de $\mathrm{P}$, aos 62 DAA, variou com a solução nutritiva, sendo que na solução $100 \%$, os teores foram numericamente maiores em Salmon Rose e menores em Orange (Tabela 3). Paradiso et al. (2003) não encontraram diferenças na absorção deste elemento entre cultivares de gérbera.

As cultivares responderam positivamente quando submetidas à solução $100 \%$, constatado pela elevação no teor e acúmulo de $\mathrm{P}$ na parte aérea, exceto para o teor de Golden Yellow e Orange, aos 62 DAA. Resultados semelhantes com aumento da concentração da solução nutritiva foram apontados por Paradiso et al. (2003) e Zheng et al. (2004), ambos para a cultura da gérbera.

Mercurio (2002) propõe o intervalo de 1,9 a 3,5 $\mathrm{g} \mathrm{kg}^{-1}$ de $\mathrm{P}$ como adequado para o desenvolvimento de plantas de gérbera. Considerando estes valores, a cultivar Cherry e a média das cultivares na solução 50\%, no período vegetativo, apresentaram valores inferiores, entretanto, sem mostrar sintomas visuais de deficiência. Esta variação possivelmente se deve à não realização de análise no período vegetativo pelo autor anteriormente citado, o qual pode ter obtido valores inferiores. Teores entre 2,3 e 3,6 $\mathrm{g} \mathrm{kg}^{-1}$ foram registrados por Bellé (1998), e entre 2,3 a 2,7 $\mathrm{g} \mathrm{kg}^{-1}$ por Savvas \& Gizas (2002) ambos ao final do período produtivo da cultura da gérbera.

De forma mais intensa que o observado para o $\mathrm{N}$, a quantidade de $\mathrm{P}$ acumulada na planta triplicou nos últimos 21 dias, portanto a maior demanda coincide com o terço final do ciclo da gérbera.

Variações no teor e acúmulo de potássio (K) foram apontadas ao longo do período produtivo (Tabela 1). Essas variações não foram encontradas por Paradiso et al. (2003), nas cultivares de gérbera, Brittani e Golden Serena.

O potássio é o nutriente exigido em maiores quantidades para culturas pertencentes à família Asteraceae (Lunt \& Kofranek, 1958; Bellé, 1998; Savvas \& Gizas, 2002; Paradiso et al., 2003), re-

Tabela 3. Interação entre cultivar e solução, para o teor de $\mathrm{N}, \mathrm{P}$ e S ( $\left.\mathrm{g} \mathrm{kg}^{-1}\right)$ aos 62 DAA e para o acúmulo de Ca (mg planta-1) aos 41 DAA. Botucatu, UNESP. 2006.

\begin{tabular}{|c|c|c|c|c|}
\hline & \multicolumn{2}{|c|}{ Teor de $\mathbf{N}$} & \multicolumn{2}{|c|}{ Teor de $\mathbf{P}$} \\
\hline & \multicolumn{4}{|c|}{ Solução (S) } \\
\hline & $50 \%$ & $100 \%$ & $50 \%$ & $100 \%$ \\
\hline Cultivar (C) & \multicolumn{4}{|c|}{$\mathbf{g ~ k g}^{-1}$} \\
\hline Cherry & $27 \mathrm{ab} B$ & 29 a A & 2,1 a $B$ & $2,9 a b A$ \\
\hline Golden Yellow & 29 a A & 29 a A & 2,5 a $A$ & $2,6 a b A$ \\
\hline Salmon Rose & 24 b $\quad B$ & 31 a $A$ & 2,3 a B & 3,1 a $A$ \\
\hline \multirow[t]{3}{*}{ Orange } & $28 \mathrm{ab} B$ & 31 a A & 2,5 a A & $2,5 \mathrm{~b} \quad \mathrm{~A}$ \\
\hline & \multicolumn{2}{|c|}{ Acúmulo de $\mathrm{Ca}$} & \multicolumn{2}{|c|}{ Teor de S } \\
\hline & \multicolumn{2}{|c|}{ mg planta $^{-1}$} & \multicolumn{2}{|c|}{$\mathbf{g ~ k g}^{-1}$} \\
\hline Cherry & $40 \mathrm{ab} A$ & 40 b A & 1,9 a $A$ & $1,5 \mathrm{~b} \mathrm{~B}$ \\
\hline Golden Yellow & $30 \mathrm{~b} \quad \mathrm{~B}$ & $40 \mathrm{~b} \mathrm{~A}$ & $1,5 \mathrm{~b} \mathrm{~A}$ & $1,6 \mathrm{~b} \mathrm{~A}$ \\
\hline Salmon Rose & 50 a $B$ & 70 a $A$ & 2,0 a $A$ & 2,1 a $A$ \\
\hline Orange & 40 ab $A$ & $40 \mathrm{~b} \mathrm{~A}$ & 2,1 a $A$ & $1,6 \mathrm{~b} \mathrm{~B}$ \\
\hline
\end{tabular}

Médias seguidas pela mesma letra não diferem entre si pelo teste de Tukey a $5 \%$, sendo minúsculas na coluna e maiúsculas na linha, entre teores e acúmulo de cada nutriente.

sultado esse confirmado também no presente trabalho. Verificaram-se acúmulos superiores para o K, justificando a importância do seu fornecimento, tanto no período vegetativo, quanto no reprodutivo, pois ambos apresentaram incrementos semelhantes (44 e 56\%, respectivamente).

Os valores do teor de K também foram elevados, estando grande parte, um pouco acima do proposto por Mercurio (2002), de 30,6 a 36,4 $\mathrm{g} \mathrm{kg}^{-1}$. Contudo, não foram observados sintomas de toxidez.

A elevação da concentração da solução nutritiva não afetou a absorção deste nutriente pela gérbera. Porém, Zheng et al. (2004) verificaram acréscimo no teor do mesmo, quando a solução nutritiva aumentou de 50\% para $100 \%$.

As respostas das cultivares dependeram das soluções, para o acúmulo de cálcio (Ca) aos 41 DAA (Tabela 3). Salmon Rose apresentou maior acúmulo tanto na solução $50 \%$ quanto na $100 \%$, apesar de não diferir significativamente de Cherry e Orange, na 50\%.Também apresentou maior acúmulo aos 62 DAA (Tabela 1). Comportamento semelhante foi observado para os teores aos 41 DAA. Lima \& Haag (1989b) verificaram em vários trabalhos que a concentração de Ca na parte aérea de crisântemo varia de acordo com a cultivar estudada.
Os valores de teor e acúmulo de Ca mostraram-se superiores quando as plantas foram fertirrigadas com a solução $100 \%$, exceto para o acúmulo aos 41 DAA, onde verificou-se que Cherry e Orange apresentaram respostas similares para as duas soluções. Neste caso, a demanda destas cultivares pode ter sido atendida com $50 \%$ da solução, pois mesmo com maiores quantidades de nutrientes disponíveis na solução completa, estes não foram absorvidos.

O acúmulo de Ca apresentou proporcionalidade nos dois períodos analisados, indicando que este nutriente deve ser fornecido igualmente durante todo o ciclo, e não reduzi-lo pela metade, conforme efetuado no presente trabalho.

As concentrações de Ca obtidas estão abaixo do limite crítico proposto por Mercurio (2002), de 16,6 $\mathrm{g} \mathrm{kg}^{-1}$. Entretanto, não foram observados sintomas de deficiência. Lima \& Haag (1989a) verificaram estes sintomas quando os teores no tecido vegetal foram de 4,6 g $\mathrm{kg}^{-1}$, para o crisântemo cultivar Golden Polaris. Bellé (1998) encontrou valores de 11 a $13 \mathrm{~g} \mathrm{~kg}^{-1}$, enquanto Savvas \& Gizas (2002) observaram uma média de $7 \mathrm{~g} \mathrm{~kg}^{-1}$. Estas variações podem estar relacionadas ao estudo de cultivares diferentes nestes trabalhos, com demandas variadas. Porém, não se descarta a possibilidade do K, cuja concentração está acima do recomendado por alguns 
autores, estar afetando negativamente a absorção de Ca.

O efeito da interação negativa entre nutrientes é bastante conhecido na literatura, pois, segundo Malavolta (1980) o excesso de potássio e/ou magnésio (Mg) pode induzir a deficiência de cálcio, pela inibição competitiva entre os mesmos. Esta observação é confirmada ao analisar o coeficiente de correlação entre os teores de $\mathrm{K}$ e Ca $\left(\mathrm{r}^{2}=-0,81\right.$ e $-0,11$, no período vegetativo e reprodutivo, respectivamente), indicando diminuição da absorção de Ca com o aumento do K. Casarini (2004) constatou essa tendência em folhas de rosa. Correlação negativa também foi observada entre $\mathrm{K}$ e $\mathrm{Mg}\left(\mathrm{r}^{2}=-0,78\right.$ no período vegetativo e -0,48, no reprodutivo). No entanto, entre Ca e Mg, esta correlação foi positiva ( $r^{2}=0,98$ e 0,77$)$.

Salmon Rose apresentou maior teor de Mg, tanto aos 41 quanto aos 62 DAA, sendo verificado também o mesmo resultado para o acúmulo. Orange teve menor taxa de acúmulo deste nutriente, aos 62 DAA (Tabela 1).

Ao final do ciclo de produção (62 DAA) verificou-se acentuado aumento nos valores de acúmulo de Mg, demonstrando a importância deste nutriente, especialmente nos últimos 21 dias.

A solução $100 \%$ contribuiu para o aumento do teor de Mg na parte aérea de plantas de gérbera, na qual os valores ficaram acima do limite crítico inferior proposto por Mercurio (2002) de 3,0 $\mathrm{g} \mathrm{kg}^{-1}$, o mesmo não ocorrendo para a solução 50\%. Valores próximos aos obtidos no presente trabalho foram registrados por Savvas \& Gizas (2002). Contudo, Zheng et al. (2004) encontraram valores superiores, de $7 \mathrm{~g} \mathrm{~kg}^{-1}$, em média.

Teores mais elevados de enxofre (S), aos 41 DAA, foram registrados em Orange sem diferir de Salmon Rose (Tabela 1), o mesmo ocorrendo com o acúmulo nesta data. Já aos 62 DAA, o comportamento das cultivares para os teores dependeu da solução nutritiva, tendo Golden Yellow menor teor na solução 50\% e Salmon Rose maior teor na 100\% (Tabela 3).

Respostas diferenciadas foram encontradas em relação às soluções nutritivas. Aos 41 DAA, as cultivares res- ponderam com aumento no teor e acúmulo de S com a solução 100\%. No entanto, aos 62 DAA, Cherry e Orange tiveram teores mais elevados deste elemento quando fertirrigadas com a solução 50\%, e as demais cultivares não mostraram diferença. Verifica-se desse modo, que no período reprodutivo, metade da solução é suficiente para atender à demanda de todas as cultivares estudadas.

Sintomas de deficiência de S em plantas de crisântemo cultivar Golden Polaris, foram verificados por Lima \& Haag (1989a) quando sua concentração no tecido foliar atingiu $1 \mathrm{~g} \mathrm{~kg}^{-1}$, teores esses inferiores aos obtidos no presente experimento.

Semelhante ao verificado para o $\mathrm{P}$, a quantidade de S acumulada triplicou no terço final, verificando-se assim, que este elemento tem importância fundamental no período reprodutivo. Apenas estes dois nutrientes não apresentaram efeito da diluição aos 62 DAA, verificada pelo aumento nos seus teores (Tabela 1) com o acréscimo da massa seca (Tabela 2), confirmando a necessidade destes, nos últimos 21 dias de ciclo da cultura da gérbera.

Com base nos resultados obtidos, conclui-se que a demanda dos macronutrientes é diferenciada entre as cultivares de gérbera, sendo desse modo, necessário que recebam manejo individualizado, para a expressão de seus potenciais genéticos. A concentração de nutrientes na solução apresentou um efeito marcante no teor e acúmulo de N, P, Ca, Mg e S na planta. Os maiores acúmulos dos macronutrientes foram registrados no terço final do ciclo de produção, de maneira mais acentuada para o fósforo e o enxofre, apresentando ao final do período produtivo, a seguinte ordem de absorção: $\mathrm{K}>\mathrm{N}>\mathrm{Ca}>\mathrm{Mg}>\mathrm{P}>\mathrm{S} \quad(415,327,33,32$, $20 \mathrm{mg}$ planta $\left.^{-1}\right)$.

\section{AGRADECIMENTOS}

Os autores agradecem às empresas Steltenpool, Sakata e Ball Van Zanten, pela contribuição indispensável no desenvolvimento da pesquisa.

\section{REFERÊNCIAS}

BELLÉ S. 1998. Sistemas de irrigação $e$ concentrações de adubação complementar na produção de Gerbera jamesonii cv 1187 em vaso. Porto Alegre: Universidade Federal do Rio Grande do Sul. 122 f. (Tese doutorado).

CAMARGO MS; CARMELLO QA; RUSCHEL J; ANTI GR. 2004. Produção de plantas e absorção de nutrientes pelo Aster ericoides (White master) cultivado em solução nutritiva com diferentes concentrações de $\mathrm{N}$ e K. Bioscience Journal 20: 83-91.

CASARINI E. 2004. Doses de $N$ e K aplicados via fertirrigação na cultura da roseira (Rosa sp) em ambiente protegido. Piracicaba: USPESALQ. 120 f. (Tese doutorado).

CHUNG YM; KIM HA; KIM KY; PARK SW; YI YB; LEE JH; KNOW OC. 2001. Morphological characteristics and genetic variation of gerbera (Gerbera hybrida Hort.). Journal of Plant Biotechnology 3:145-149.

FANELA TLM; MOTA PRD; VILLAS BÖAS RL; LUDWIG F; FERNANDES DM. 2006. Influência de diferentes níveis de tensão de água na cultura da gérbera desenvolvida em substrato. In: MOSTRA CIENTÍFICA EM CIÊNCIAS AGRÁRIAS, 2.; MOSTRA CIENTÍFICA DA FMVZ, 10.; REUNIÃO CIENTÍFICA EM CIÊNCIAS AGRÁRIAS DO LAGEADO, 13. Resumos... Botucatu: UNESP (CD-ROM).

KÄMPF AN. 2000. Produção comercial de plantas ornamentais. Guaíba: Agropecuária. 254 p.

LIMA AMLP; HAAG HP. 1989a. Nutrição mineral de plantas ornamentais. XII. Deficiências de N, P, Ca, Mg, S e B em crisântemo (Chrysanthemum morifolium cV Golden Polaris). In: HAAG HP; MINAMI K; LIMA AMLP (eds). Nutrição mineral de algumas espécies ornamentais. Campinas: Fundação Cargill, p. 110-132.

LIMA AMLP; HAAG HP. 1989b. Nutrição mineral de plantas ornamentais. XIII. Absorção de macronutrientes pelo crisântemo, cultivar Golden Polaris. In: HAAG HP; MINAMI K; LIMA AMLP (eds). Nutrição mineral de algumas espécies ornamentais. Campinas: Fundação Cargill, p. 64-102.

LUNT OR; KOFRANEKAM. 1958. Nitrogen and Potassium nutrition of Chrysantemum. Proceedings of the American Society for Horticultural Science 72: 487-497.

MALAVOLTA E. 1980. Elementos de nutrição mineral de plantas. São Paulo: Agronômica Ceres. 253 p.

MALAVOLTA E; VITTI GC; OLIVEIRA SA. 1997. Avaliação do estado nutricional das plantas: princípios e aplicações. Piracicaba: Potafos. 319 p.

MERCURIO G. 2002. Gerbera cultivation in greenhouse. The Netherlands: Schreurs. 206 p.

PARADISO R; DE PASCALE S; APREA F; BARBIERI G. 2003. Effect of electrical conductivity levels of nutrient solution on growth, gas exchanges and yield of two gerbera cultivars in soilless system. Acta Horticulturae 609: 165-171.

RADICE S; MARCONI PL. 1998. Clonación in 
vitro de diversas cultivares de Gerbera jamesonii a partir de capítulos florales. Revista de la Facultad de Agronomía, La Plata 103: 111-118.

ROGERS MN; TJIA BO. 1990. Gerbera production. Timber Press Growers handbook series 4: $116 \mathrm{p}$.

SAVVAS D; GIZAS G. 2002. Response of hydroponically grown gerbera to nutrient solution recycling and different cation ratios. Scientia Horticulturae 96: 267-280.
STRINGUETA AC; MARTINEZ HP; CARDOSO AA; COSTA CA. 2003. Teores foliares de macronutrientes em crisântemos cultivados em substratos contendo composto de lixo urbano e casca de arroz carbonizada. Revista Brasileira de Horticultura Ornamental 9: 191-197.
ZHENG Y; GRAHAM T; RICHARD S; DIXON M. 2004. Potted gerbera production in a subirrigation system using low-concentration nutrient solutions. HortScience 39: 1283-1286. 